\title{
Der Schutzauftrag des Rechts - im Lichte der 70. Staatsrechtslehrertagung 2010 in Berlin
}

\author{
Helmuth Schulze-Fielitz"
}

Unter dem sehr offenen Rahmenthema „Der Schutzauftrag des Rechts“ widmete sich die 70. Staatsrechtslehrertagung vom 22. bis 24. September 2010 in Berlin - jubiläumsgerecht an der Humboldt-Universität - vier Problemfeldern: dem (Grundrechts-)Schutz der Privatheit, der Verteilungsgerechtigkeit im Gesundheitswesen, dem Wandel von Verwaltungsverfahren in ihrem Eigenwert und dem Regulierungsrecht, nun speziell aus der Perspektive des Verbraucherschutzes. Eine derartige Zusammenführung von anscheinend Disparatem spiegelt aktuelle Wandlungen in der Staatsrechtslehre als Wissenschaft und ihren Gegenstandsbereichen, die sich etwa unter den Gesichtspunkten einer Pluralisierung der Arten des Problemzugangs oder der intradisziplinären Ausweitung des Forschungsrahmens deutlich an den acht Vorträgen ablesen ließen.

1. So verdeutlichten schon die ersten beiden Referate zum „Grundrechtsschutz der Privatheit" eine wohl zunehmende Pluralisierung juristischer Denkstile, sobald man sich wissenschaftlich nicht mit einem insoweit vereinheitlichenden Rechtsprechungspositivismus begnügen will. Martin Nettesheim (Tübingen) begann mit einem HegelZitat und auf großgesellschaftstheoretischem Niveau, indem er dem Voyeurismus als zentraler kultureller Erscheinung der heutigen Gesellschaft die gegenständliche, räumliche oder soziale Privatheit gegenüberstellte, die sich in Abgrenzung zu Staat, Öffentlichkeit oder Markt und deren Erzeugung von „Gegenbildern“ konstituiere und seit ihrer Politisierung in den 1960er und 1970er Jahren als Aufgabe des Rechts und Produkt der Politik zu begreifen sei. Die verfassungsgerichtliche Judikatur sei ihr teilweise nicht gewachsen, namentlich bei Formen der „staatlichen Verbildlichung“ (etwa durch Rasterfahndung, Videoüberwachung öffentlicher Plätze), weil dabei das Verständnis vom Grundrechtseingriff (schon bei einem „sich einstellenden Gefühl des Überwachtwerdens“) überdehnt werde, aber auch bei den Formen der Verbildlichung durch private Dritte. Sein rechtsdogmatischer „freiheitsakzessorischer Neuansatz“ setzt bei der Erstellung und Nutzung der Gegenbilder einer Person an und formuliert in diesem Lichte je nach Freiheitsrecht differenzierte Anforderungen einerseits an den Staat, andererseits horizontal an private Dritte bei der Erhebung und Verwendung von Daten und der Nutzung der Gegenbilder; dadurch soll ein einheitliches Grundrecht auf informationelle Selbstbestimmung entbehrlich werden. Demgegenüber machte Oliver Diggelmann (Zürich) auf einer mittleren Abstraktionsebene verschiedene privatheitsgefährdende Entwicklungen in Technik, Wissen,

* Prof. Dr. Helmuth Schulze-Fielitz war Inhaber des Lehrstuhls für Öffentliches Recht, Umweltrecht und Verwaltungswissenschaften an der Juristischen Fakultät der Bayerischen Julius-Maximilians-Universität Würzburg. 
Ökonomie und Wertebasis zum Ausgangspunkt seiner Überlegungen, verglich sodann die Schutzkonzepte von EMRK und US-Verfassung im Blick auf Gemeinsamkeiten (Schutz der Unverletzlichkeit der Wohnung und der vertraulichen Kommunikation), aber auch auf rechtskulturell begründete Unterschiede (beim Schutz der „öffentlichen Privatheit“ oder bei der Reichweite der selbstbestimmten Lebensführung), ehe er in einem dritten Schritt ausgewählte dogmatische Schlüsselfragen identifizierte: die Abnahme der begrenzenden Kraft des Verhältnismäßigkeitsprinzips, problematische Folgen staatlicher Heimlichkeit für Rechtsschutz und Konformitätsdruck sowie eine Aktualisierung der Schutzpflichten des Gesetzgebers aufgrund der Kommerzialisierung vertraulicher Personendaten, allgemein im Wirtschaftsverkehr, besonders im Bereich Massenmedien. - So sehr die beiden - in einem traditionellen Sinne gleichermaßen rechtsdogmatisch akzentuierten - Analysen sich sei es empirischer, sei es gesellschaftlicher Hintergrundentwicklungen ausdrücklich zu vergewissern suchten - das war früher auch nicht immer selbstverständlich -, so sehr unterschieden schon sie sich in der theoretischen Abstraktionshöhe des Problemzugangs, in der Art der Problemabschichtung, in der pragmatischen Rückbindung an die Perspektiven des juristischen Alltags und in dem Vertrauen in die zukünftige Leistungsfähigkeit des bestehenden rechtsdogmatischen Instrumentariums.

2. Auch die Vorträge zum zweiten Thema („Knappheit und Verteilungsgerechtigkeit im Gesundheitswesen“) waren arbeitsteilig aufeinander abgestimmt und bildeten so eine große Bandbreite öffentlichrechtlicher Problemzugänge ab. Joachim Lege (Greifswald) betrachtete die Verteilungskämpfe im Gesundheitssystem in der rechtsphilosophisch inspirierten Perspektive einer gerechten Verteilung der „Gesundheitsgüter" - sei es an den Grenzen der Sektoren der gesetzlichen und privaten Krankenversicherung bei der Verteilung der "guten Risiken“, sei es bei der Verteilung unter den verschiedenen Gruppen der Leistungserbringer in einem „neofeudalen System der Beuteverteilung auf Gegenseitigkeit“. Er beschrieb in mitunter plastischen $\mathrm{Zu}$ spitzungen das Gesundheitssystem als eines mit „kleinen Ungerechtigkeiten“, bei denen das Nebeneinander von privater und von gesetzlicher Krankenversicherung nicht zu rechtfertigen sei, auch nicht die Abkoppelung der Verteilungsgerechtigkeit von der Erwirtschaftungsgerechtigkeit oder die Intransparenz der Verteilung von Risiken. Seine Verbesserungsvorschläge im Namen von sozialstaatlicher Gleichheit und Fürsorge beschränkten sich weithin auf Tendenzaussagen, ohne die Grundmodelle zur Reform der Finanzierung des Gesundheitswesens ( „Bürgerversicherung “ vs. „Kopfpauschale“) verfassungsrechtlich infrage zu stellen. Demgegenüber richtete sich Thorsten Kingreen (Regensburg) von vornherein stärker in der einfachgesetzlichen Systematik des Sozialrechts im Blick auf die Lösungsstrategien für Probleme im „Knappheitslaboratorium Gesundheitswesen“ ein, die der Gesetzgeber weder durch bloße Rationalisierung noch ohne konkrete Regelungen der Rationierung und Priorisierung von Leistungen lösen könne. De lege lata erfolge die Notwendigkeit der 
Krankenbehandlung nach Maßgabe von Richtlinien des Gemeinsamen Bundesausschusses aus Vertretern von Krankenkassenverbänden und Leistungserbringern. Dabei erlaube der juristische Begriff der Krankheit nach Maßgabe einerseits der Eigenverantwortung der Versicherten und der Zumutbarkeit, andererseits nach Wirtschaftlichkeitsüberlegungen den Ausschluss von Versicherungsleistungen, wie sie auch bei den Kosten-Nutzen-Bewertungen von Arzneimitteln impliziert seien. Verlangt sei ein parlamentarisches Gesetz über die wesentlichen Präferenz- und Verteilungsentscheidungen und über die Organisations- und Verfahrensregelungen für die normativ (politisch, ethisch) entscheidenden Entscheidungsträger wie den Gemeinsamen Bundesausschuss, der deshalb bislang unzureichend demokratisch legitimiert sei. Eine Rationierung von Gesundheitsleistungen verlange eine Politisierung der Entscheidungen im Vorfeld parlamentarischer Gesetze und - zu reaktivierender Rechtsverordnungen. - Diese Art der sich wechselseitig ergänzenden Problembehandlung durch beide Referenten hat zwar den Nachteil, dass manchen fachlichen Spezialisten der Problemzugang teils zu wenig bzw. zu sehr sozialrechtlich oder umgekehrt zu sehr oder zu wenig allgemein-philosophisch erscheinen konnte. Der große Vorteil liegt darin, dass sachlich Perspektiven auf unübliche Weise verklammert werden und personell auch Nichtspezialisten als Zuhörer auf ein Forschungsfeld geführt werden, mit dem sie sich ohne den „Zwang“ zur Teilnahme an einer Plenarveranstaltung nicht so schnell ausgesetzt hätten. Der generelle wissenschaftspolitische mainstream (etwa auch in der Deutschen Forschungsgemeinschaft), inter- und intradisziplinäres Zusammenwirken über die eigenen engeren Fachgrenzen hinaus wegen der (vermutlich) innovationsfördernden Anregung zu wissenschaftlichen Transferüberlegungen zu fordern und zu fördern, wird durch solche Themenstellungen im Plenum (unter Verzicht auf sonst ubiquitäre Veranstaltungen für Spezialisten etwa in Sektionen) wie von selbst ermöglicht. Zugleich kann so u.U. den Gefahren einer Abkopplung von Teilrechtsgebieten mit Sonderdogmatiken von den allgemeinen Entwicklungen der wissenschaftlichen Systembildung begegnet werden.

3. In auf den ersten Blick traditionelleren Bahnen bewegt sich die Frage nach dem „Eigenwert des Verfahrens im Verwaltungsrecht“. Elke Gurlit (Mainz) rekapitulierte den Verfahrensgedanken in der jüngeren deutschen Entwicklung des Verwaltungsrechts, präzisierte die Funktionen des Verwaltungsverfahrens unter Überwindung der Entgegensetzung von dessen „Eigenwert“ vs. „dienender Funktion“ und stellte die Eigenständigkeit des Verfahrens als relatives Medium der Richtigkeitsgewähr in den Kontext der Steuerungskraft durch das materielle Recht, ohne dabei grundrechtliche und partizipatorische Interessen oder Gesichtspunkte wie die Transparenz der Entscheidungsfindung und Entscheidungsakzeptanz zu vernachlässigen. Deren relatives Gewicht wurde differenziert im Blick auf einige entscheidungsbezogene Verfahrenstypen erläutert - mit Skepsis etwa gegenüber spezifisch wissensgenerierenden Verfahren ohne freien Zugang externen Sachverstandes oder teilweise 
auch gegenüber Verfahren, in denen Sachentscheidung und Verfahren zu stark entkoppelt werden. Die differenzierte Behandlung der Folgen von Verfahrensfehlern (mit Kritik an $\$ 46 \mathrm{VwVfG}$ ) war vom Grundgedanken geleitet, dass gerichtliche Kontrollverfahren Verwaltungsverfahren wegen deren Eigenwert nicht umstandslos ersetzen können. Das nur beiläufig in Bezug genommene „Modernisierungspotential" des Europarechts wurde im folgenden, seinem Schwerpunkt nach rechtsvergleichenden Referat von Michael Fehling (Hamburg) deutlich korrigiert. Der rechtsvergleichende Blick in die USA, England, Frankreich und nach Europa relativierte manche neuerdings verbreitete Pauschalannahme: Die Reichweite von Anhörungsrechten und Begründungspflichten etwa sei im Ausland nicht größer; auch dort gibt es funktionale Äquivalente für Heilungs- und Unbeachtlichkeitsregeln, die nur wegen des weit größeren Entscheidungsspielraums der Verwaltung weniger zum Zuge kämen. Auch dort gibt es keinen isolierten Rechtsschutz gegen vorbereitende Verfahrenshandlungen, und der teils weitergehende Individualrechtsschutz werde teilweise relativiert. Eine Kompensation der geringeren materiellen Kontrolldichte durch weitreichende Verfahrensgarantien gelte nur bei komplexen Verwaltungsentscheidungen, deren selektive Betrachtung keinen Rückschluss auf einen generell höheren Eigenwert des Verwaltungsverfahrens in anderen Rechtsordnungen erlaube. Der Eigenwert des Verwaltungsverfahrens diene etwa zur Rechtswahrung, zur frühzeitigen Problemsensibilisierung, im Vergabeverfahren zu chancengleichem Wettbewerb, in gestuften Verbundverfahren zur kommunikativen und effektiven Durchsetzung des Unionsrechts oder zur Verfahrensbeschleunigung - die Aufwertung des Verfahrensgedankens sei dementsprechend gesamteuropäischer Natur, weil sie mit einer stärkeren Interessenverarbeitung den Eigenwert des Verwaltungsverfahrens erhöhen und insoweit der Verwirklichung des materiellen Rechts dienen könne, ohne dass für das deutsche Verfahrensrecht insoweit ein struktureller Rückstand kennzeichnend sei. Was für die eher überschaubare Zahl der Rechtsvergleicher im Verwaltungsrecht weniger aufregend erscheinen mochte, war für den eher national „introvertierten“ Verwaltungsrechtler ausgesprochen erhellend, weil es so deutlich vorherrschenden Argumentationstrends widersprach, die im vergangenen Jahrzehnt einflussreich geworden sind. Die Internationalisierung und Europäisierung der wissenschaftlichen Diskussionen wirkt sich so immer unumgänglicher und erkennbarer unmittelbar auf den Stand der wissenschaftlichen Diskussion auch im insoweit scheinbar selbständigen nationalen Verwaltungsrecht aus.

4. Solches wird erst recht dort sichtbar, wo wie beim „Schutz der Verbraucher durch Regulierungsrecht“ ein neues Rechtsgebiet in Bezug genommen wird, dessen Existenz - mag sie auch mitunter noch bezweifelt werden ${ }^{1}$ - sich jedenfalls wesentlich europäischen Impulsen verdankt. Johannes Hellermann (Bielefeld) nahm die spezi-

1 Vgl. H.-W. Laubinger, Regulierungsrecht: Gibt's das - und wenn ja: wie viele?, VBlBW 2010, S. 306 ff., zu M. Fehling / M. Ruffert (Hrsg.), Regulierungsrecht, 2010. 
fisch aufgegebene Perspektive des Verbraucherschutzes im Dreieck von regulierendem Staat, Unternehmen und Verbrauchern unter den Bedingungen von Marktversagen ernst und ordnete den europäisch definierten „Diensten von allgemeinem wirtschaftlichen Interesse“ insoweit grundlegende Bedeutung für den Schutz der Verbraucher zu. Dabei sprengt die charakteristische Gemengelage von öffentlichrechtlichen und privatrechtlichen Normen die herkömmliche Betrachtung nach Rechtsgebieten, weil früher leistungsverwaltungsrechtlich geregelte Beziehungen heute einerseits privatrechtlich geregelt, andererseits öffentlich-rechtlich überlagert werden. Die einzelnen einfachgesetzlichen Verbraucherschutzziele gestalten die dichten, vor allem sekundärrechtlichen europäischen Vorgaben für das Regulierungsrecht aus und prägen letztlich die Durchsetzung der Verbraucherschutzinteressen auch in den privatrechtlichen Kundenbeziehungen. Ungeachtet des dominierenden Leitbilds des mündigen und umsichtig handelnden Verbrauchers verlange Regulierungsrecht vor dem Hintergrund störender Marktunvollkommenheiten einen über den Ausgleich von Informationsasymmetrien und Kundenschutz hinausgehenden spezifischen Verbraucherschutz, insbesondere in den Netzwirtschaften mit ihren weiterhin wirksamen natürlichen Monopolen. Dennoch soll es kein Regulierungsverwaltungsrecht als dritte Säule neben der Ordnungs- und Leistungsverwaltung geben, weil Regulierungsrecht quer zu den einzelnen Verwaltungsaufgaben und zu den einzelnen Rechtsgebieten liege. Demgegenüber stellte der Zweitreferent Wolfgang Durner (Bonn) vielschichtig sehr grundsätzliche Vorbehalte gegen europäische Tendenzen allgemein - nicht nur, aber auch auf dem Gebiet des Verbraucherschutzes - in den Vordergrund. Empirisch und analytisch kritisierte er die Unschärfe der Begriffe „Regulierungsrecht" und „Verbraucherschutzrecht“, sah in Regulierungsverwaltung erhebliche politische Gewichtsverschiebungen zugunsten der unabhängigen Regulierungsbehörde und des Einflusses der EU-Kommission zulasten der Rückkopplung an die Regierung und der gerichtlichen Kontrolle. Er diagnostizierte generell ein Vordringen exekutivischer Machtkonzentration, die auch im Sinne einer paternalistischen Verbraucherpolitik in einem - bald möglicherweise voll ausgebildeten - Regulierungverbund instrumentalisiert werde, schon weil die Eigeninteressen der europäischen Exekutive nach neuen Tätigkeitsfeldern mit breiten Gestaltungsspielräumen drängten. Dagegen wurden die rechtsstaatlich-demokratischen Strukturprinzipien des Grundgesetzes und des Unionsvertrages in Stellung gebracht: etwa die grundrechtlichen Abwehrrechte der Regulierten; die Forderung nach materiellem Rechtsschutz über den Maßstab einer bloßen Abwägungskontrolle hinaus; nach Präzisierung von Eingriffszielen statt rechtsstaatlich unzulässiger Blankettermächtigungen zur Verfolgung des unbestimmten Zieles der „Wahrung der Verbraucherinteressen“ oder nach politischer Legitimation und Verantwortung (statt Entpolitisierung durch institutionell unabhängige, nur scheinbar neutrale Experten) im Sinne parlamentarischer Input-Legitimation in Rückkoppelung zum Bürger statt einer bloß (mit Art. 20 GG 
unvereinbaren) Output-Legitimation am Maßstab der Wohltaten für den Verbraucher. - Diese meinungsstarken Thesen auf der Linie einer fast schon traditionellen „Bonner“ Europaskepsis provozierten entsprechende Kritik, verdeutlichten aber einmal mehr die Verwobenheit der nationalen mit der europäischen Rechtslage, die schwindende Problemlösungskraft nicht nur dieser Zäsur, sondern auch einer Grenzziehung zwischen Öffentlichem Recht und Privatrecht, und letztlich die Durchdringung rechtlicher Argumentation mit sozialwissenschaftlichen Befunden oder Hypothesen: Die systematischen Grenzziehungen in der Staatsrechtslehre werden allenthalben unübersichtlicher.

5. Die Tagungsfrage nach dem Schutzauftrag „des Rechts“ allgemein, über das Öffentliche Recht hinaus, spiegelt solche übergreifenden Perspektiven ebenso wie der Umstand, dass die jahrzehntelange Tagungspraxis der thematischen Gliederung in eine verfassungsrechtliche Fragestellung am ersten und eine verwaltungsrechtliche Schwerpunktsetzung am zweiten Verhandlungstag mittlerweile nur noch grob erkennbar ist, weil seit längerem nicht Rechtsgebiete des Öffentlichen Rechts und ihre rechtsdogmatischen Einzelfragen, sondern (auch) sachliche Problemfelder themensetzend sind, auf denen in aller Regel europa-, verfassungs- und verwaltungsrechtliche Zusammenhänge verknüpft sind. Hinzu kommen die für eine sachgerechte Systematisierung notwendigen Grenzüberschreitungen hin zu den empirischen Grundlagen der Rechtsdogmatik des Öffentlichen Rechts und die verstärkte Notwendigkeit einer Vergewisserung der alltagspraktischen Ebene mit größeren Entwicklungszusammenhängen im Öffentlichen Recht, wohl auch im Zusammenspiel mit dem Privatrecht und mit nichtrechtlichen funktional äquivalenten Regelungs- und Steuerungsformen. Solche Trends können nicht auf der Ebene etwa verwaltungsgerichtlicher Einzelfallentscheidungen bei der Gesetzesanwendung erkannt werden, sondern nur durch eine übergreifende wissenschaftliche Distanznahme. Hierin liegt ein zentrales Spezifikum von Staatsrechtslehrertagungen. Die damit einhergehende Vervielfältigung und Heterogenisierung von Problemstellungen ist eine wachsende wissenschaftliche Herausforderung, die vielleicht durch eine gewisse Starre des klausurorientierten Ausbildungskanons in der Universitätspraxis des Alltags (und der korrespondierenden Ausbildungsliteratur) eher verdeckt wird.

Der Verbreiterung des Themenzuschnitts bei den Referaten entspricht eine Verbreiterung der möglichen wissenschaftlichen Diskursgegenstände. Die Diskussion war denn auch weniger durch - angesichts des arbeitsteiligen Zusammenwirkens bis an die Grenze zur Langeweile minimierte - sachliche Kontroversen zwischen den Referenten als durch den Widerspruch aus dem Plenum zu besonders schneidig vorgetragenen Thesen bestimmt. Täuscht der subjektive Eindruck, dass die einzelnen Referate auch eine größere Bandbreite ihrer Bewertung (im Plenum und beim Kaffee) fanden als bei geschlossenen Fachzirkeln gemeinhin zu erwarten ist? Der Pluralisie- 
rung der juristischen Problemzugänge scheint eine Pluralisierung wissenschaftlicher Erwartungshorizonte in der Zuhörerschaft zu entsprechen. Vielleicht geht dabei die gemeinsame Sprache der Staatsrechtslehre verloren ${ }^{2}$. Aber die Wissenschaft des Öffentlichen Rechts muss sich doch wohl primär an den durch den Wandel der Erscheinungsformen von Staatlichkeit hervorgerufenen neuartigen Problemen und ihrer Lösung orientieren, nicht umgekehrt, und sie muss ihren Anspruch pflegen, durch Binnenrechtsvergleichung, zumindest auf Basis des Grundgesetzes, eine Einheit des Öffentlichen Rechts und seiner wissenschaftlichen Systematisierung ungeachtet aller zentrifugalen Kräfte zu wahren oder immer wieder neu herzustellen. Das wird zumindest gefördert, wenn die Form der Tagungsorganisationen weiterhin durch gemeinsame Diskussionen in Plenarveranstaltungen erhalten bleibt, und wenn die individuellen Neigungen zur Bequemlichkeit durch eine zu enge fachliche Spezialisierung in der eigenen Veröffentlichungspraxis, die ohne Rückbindung ans Allgemeine schnell den Wald vor lauter Bäumen nicht mehr zu sehen droht, nicht als vorbildhaft für den wissenschaftlichen Fortschritt angesehen werden. Die Jahrestagung 2010 hat auch solche Aufgaben für die Staatsrechtslehre als Wissenschaft des Öffentlichen Rechts wie für den einzelnen Wissenschaftler einmal mehr plastisch vor Augen geführt.

2 Vgl. J. Isensee, Staatsrechtslehre als Wissenschaft, JZ 2009, S. 949 (951): „Die babylonische Sprachverwirrung, die zwischen den verschiedenen Wissenschaften herrscht, ist inzwischen auch in das Internum der Staatsrechtslehre eingezogen “. 